ARTICLE

https://doi.org/10.1038/s41467-019-11382-8

\title{
Development of bifunctional organocatalysts and application to asymmetric total synthesis of naucleofficine I and II
}

Yong-Hai Yuan", Xue Han¹, Fu-Ping Zhu' ${ }^{1}$, Jin-Miao Tian², Fu-Min Zhang ${ }^{1}$, Xiao-Ming Zhang ${ }^{1}$, Yong-Qiang Tu,2, Shao-Hua Wang (iD ${ }^{1} \&$ Xiang Guo ${ }^{1}$

The proline-type organocatalysts has been efficiently employed to catalyze a wide range of asymmetric transformations; however, there are still many synthetically useful and challenging transformations that remain unachievable in an asymmetric fashion. Herein, a chiral bifunctional organocatalyst with a spirocyclic pyrrolidine backbone-derived containing fluoroalkyl and aryl sulfonamide functionalities, are designed, prepared, and examined in the asymmetric Mannich/acylation/Wittig reaction sequence of 3,4-dihydro- $\beta$-carboline with acetaldehyde, acyl halides, and Wittig reagents. As a result, the spirocyclic pyrrolidine trifluoromethanesulfonamide catalyst can facilitate this versatile sequence as demonstrated by 18 examples displaying excellent enantioselectivity (up to $94 \%$ ee), as well as moderate to good yields (up to $54 \%$ over 3 steps). As a practical application, the asymmetric total synthesis of naucleofficine I (1) and II (1) in ten steps have been accomplished.

\footnotetext{
${ }^{1}$ State Key Laboratory of Applied Organic Chemistry and College of Chemistry and Chemical Engineering, Lanzhou University, Lanzhou 730000, P. R. China. ${ }^{2}$ School of Chemistry and Chemical Engineering, Shanghai Jiao Tong University, Shanghai 200240, P. R. China. Correspondence and requests for materials should be addressed to Y.-Q.T. (email: tuyq@lzu.edu.cn)
} 
T he metal-free organocatalysis based on the dual activation of amino acids and their analogs has been displaying versatile utilities in the synthesis of natural products and pharmaceuticals ${ }^{1-5}$. The proline-type organocatalysts represent a large class of useful bifunctional catalytic systems, which have facilitated a wide range of asymmetric reactions of carbonyls, imines, and their variants, including aldol, Michael addition, Diels-Alder cycloaddition, Mannich reaction, and so on ${ }^{6-12}$. Despite these advances, there are still many inspiring transformations that remain unachievable in asymmetric synthesis. Therefore, development of more and reliable bifunctional organocatalysts is of high demand for effecting these transformations. Aimed at the asymmetric synthesis of biologically important natural products and drug molecules, our current efforts have been focused on developing novel and effective catalysis systems to enable synthetically challenging transformations so as to explore the concise synthetic approaches. As indicated in Fig. 1, at least four kinds of different natural products, the naucleofficine I (1a) and II (1b), yohimbine, and berbanes, contain the common complex $N$-fused bicyclic structures 6 with multi-substituents and stereocenters. Compounds $\mathbf{1 a}$ and $\mathbf{1 b}$ are isolated from Nauclea genus and exhibit antibacterial and antiviral biological activities $^{\mathrm{P} 3,14}$. They represent the characteristic structures of a number of monoterpenoid indole alkaloids and have not been synthesized so far, although some approaches to their analogs have been reported by employing free radical cyclization and the Pictet-Spengler reaction as key steps ${ }^{15-18}$.

In previous reports, the the spirocyclic pyrrolidine (SPD) backbone-derived organocatalysts have displayed high enantioselectivity in several reactions, possibly due to the rigid chiral environment of quaternary carbon stereocenter and spirocylic scaffold, and the flexibility of the lone electron pair of the pyrrolidine moiety ${ }^{19-21}$. In this regard, it is hypothesized that attachment of the strong acidic fluoroalkylsulphonamide motif to a sterically hindered SPD backbone would generate a new bifunctional catalyst, which would exhibit stronger acidity and superior enantio-induction ability compared with proline but still maintain the active pyrrolidine amine. Furthermore, the BINOLphosphonyl TfNH catalyst (Table 1, Cat 8) exhibits much stronger acidity than BINOL-phosphoric acid alone, due to its stability of counter anion and strong electron-accepting property of $\mathrm{Tf}$ group ${ }^{22-25}$. Herein, we present our research results on the asymmetric Mannich/acylation/Wittig sequence to efficiently assemble the tetrahydro- $\beta$-carboline $\mathbf{5}$, which allows us to accomplish the total synthesis of naucleofficine I (1a) and naucleofficine II (1b) in a concise way.

\section{Results}

Design plan. The synthesis of natural products in Fig. 1 could be simplified to establishment of the single $\mathrm{C} 3$ stereocenter and installation of two unsaturated chains in 5. In this work, we focus on the synthesis of the alkaloids $\mathbf{1 a}$ and $\mathbf{1 b}$, our retro-synthesis involves alternatively a late-stage $O$-hetero-Diels-Alder cycloaddition to construct the $\mathrm{D} / \mathrm{E}$ rings with multi-functional groups and stereogenic centers in one step from the key intermediate $\mathbf{5}$ (Fig. 1). Therefore, we envision a versatile Mannich/acylation/ Wittig sequence would enable the construction of 5 from commercially available 3,4-dihydro- $\beta$-carboline 2 , acetaldehyde, acyl halides, and corresponding Wittig reagents. To achieve this catalytic asymmetric sequence, however, some challenges exist, including the following: (1) the starting $\mathrm{N}$-aliphatic imine is relatively inactive compared with generally used active Boc- $-26,27$, Ts- ${ }^{28,29}$, aryl-imine species ${ }^{30,31}$, and $\mathrm{Cbz}_{-}{ }^{32}$, which requires a stronger acidity to activate the $\mathrm{C}=\mathrm{N}$ bond via hydrogen bonding; (2) the active acetaldehyde ${ }^{33-39}$ could easily form byproducts via aldol condensation; and (3) asymmetric Mannich reaction of 3,4-dihydro- $\beta$-carboline and aldehydes is a challenging work, which has not been reported up to now, maybe due to the instability of the resulting amino aldehyde.
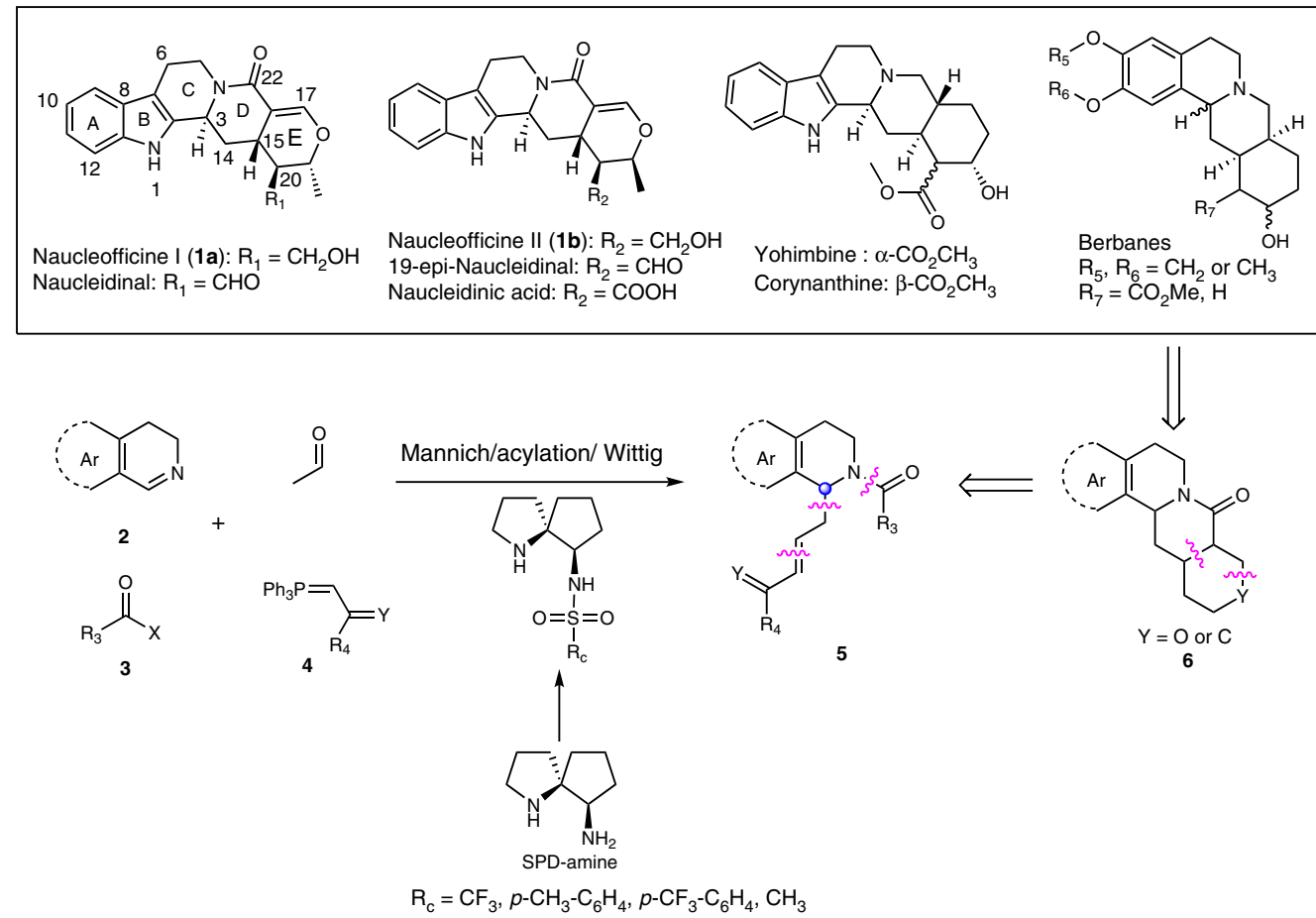

Fig. 1 Design of asymmetric synthesis of monoterpenoid alkaloids. Retro-synthesis involves Diels-Alder reaction and Mannich/acylation/Wittig sequent reaction 

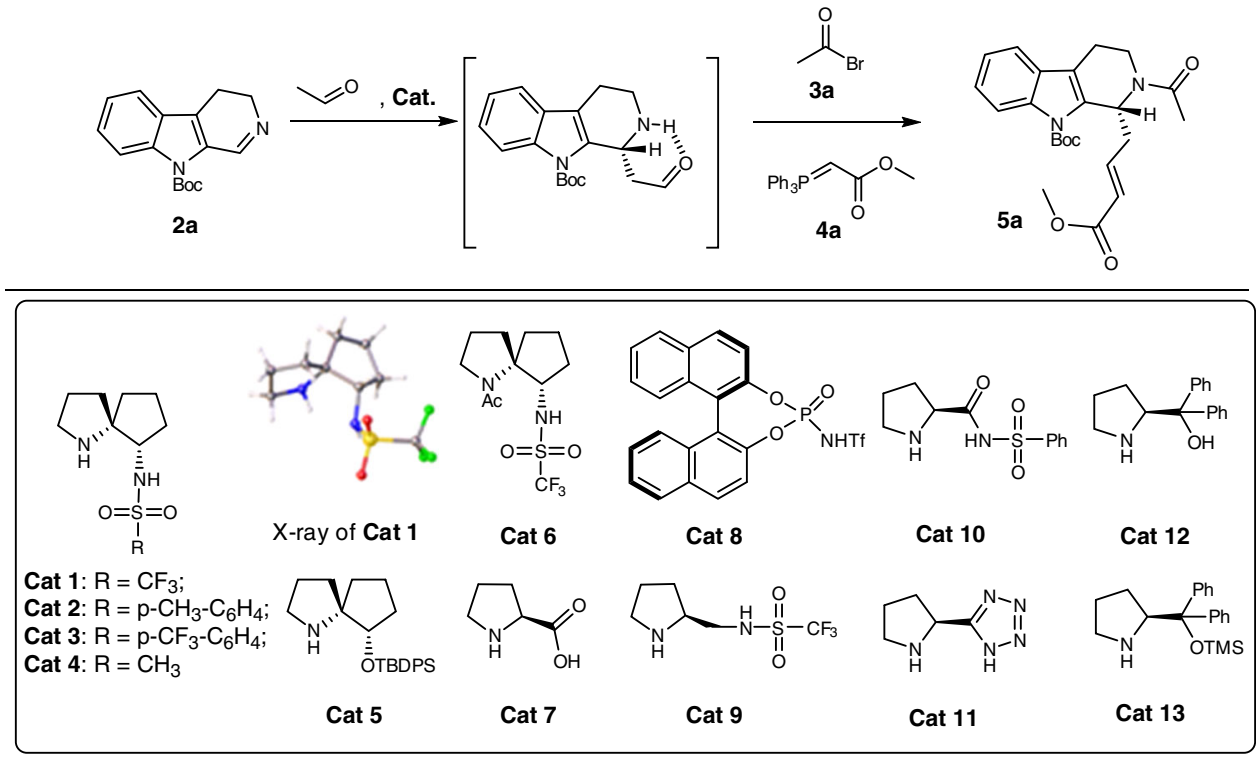

\begin{tabular}{|c|c|c|c|c|c|c|}
\hline Entry & Catalyst & $T$ [으] & Additive & $t$ [h] & Yield [\%] ${ }^{\mathbf{b}}$ & ee $[\%]^{c}$ \\
\hline 1 & Cat 1 & 0 & - & 8 & 21 & 51 \\
\hline 2 & Cat 1 & 0 & $\mathrm{PhCO}_{2} \mathrm{H}$ & 8 & 8 & -1.1 \\
\hline 3 & Cat 1 & 0 & PTS & 8 & 12 & -57 \\
\hline 5 & Cat 1 & 0 & DMAP & 20 & 29 & 87 \\
\hline 6 & Cat 1 & 0 & DBU & 20 & 47 & 86 \\
\hline 7 & Cat 1 & -5 & $\mathrm{Et}_{3} \mathrm{~N}$ & 10 & 48 & 88 \\
\hline 10 & Cat 3 & 0 & $\mathrm{Et}_{3} \mathrm{~N}$ & 48 & 12 & 8 \\
\hline 11 & Cat 4 & 0 & $\mathrm{Et}_{3} \mathrm{~N}$ & 48 & $<5$ & 32 \\
\hline 12 & Cat 5 & 0 & $\mathrm{Et}_{3} \mathrm{~N}$ & 48 & $<5$ & 18 \\
\hline 13 & Cat 6 & 0 & $\mathrm{Et}_{3} \mathrm{~N}$ & 48 & $<5$ & 14 \\
\hline 14 & Cat 7 & 0 & $\mathrm{Et}_{3} \mathrm{~N}$ & 48 & $\mathrm{ND}^{\mathrm{d}}$ & $\mathrm{ND}^{\mathrm{d}}$ \\
\hline 15 & Cat 8 & 0 & $\mathrm{Et}_{3} \mathrm{~N}$ & 30 & 15 & 20 \\
\hline 16 & Cat 9 & 0 & $\mathrm{Et}_{3} \mathrm{~N}$ & 6 & 26 & 68 \\
\hline
\end{tabular}

a Unless otherwise noted, all reactions were carried with $\mathbf{2 a}(0.1 \mathrm{mmol})$, acetaldehyde $(0.3 \mathrm{mmol})$, catalyst $(0.02 \mathrm{mmol})$, and additive $(0.05 \mathrm{mmol})$, in $0.5 \mathrm{~mL} D C E$ and $0.5 \mathrm{~mL} \mathrm{H}_{2} \mathrm{O}$; after the reaction is complete, the solution was evaporated, then DCM $(1 \mathrm{~mL}), \mathrm{K}_{2} \mathrm{CO}_{3}(0.2 \mathrm{mmol})$, acyl halide $\mathbf{3}(0.15 \mathrm{mmol})$, and Wittig reagent $4(0.2 \mathrm{mmol})$ was added sequentially to the reaction system

b Isolated yield over three steps

c Determined by HPLC analysis

d No reaction detectable

DBU 1,8-diazabicyclo[5.4.0]undec-7-ene, DMAP 4-dimethylaminopyridine, PTS p-toluenesulfonic acid, DCE 1,2-dichloroethane, DCM dichloromethane

Bold values represent the optimal condition

Reaction condition optimization. Initially, four catalysts Cat 1-4 with substituents possessing different electronic properties were prepared from (S,S)-SPD-amine (for details, see Supplementary Note 2). Imine 2a was selected as a model substrate to screen the optimal condition of the Mannich sequence with acetaldehyde, acetyl bromide, and methyl(triphenylphosphoranylidene)-acetate. Fortunately, the expected Mannich sequence could take place in the presence of Cat 1 in $D C E / H_{2} \mathrm{O}$ at $0{ }^{\circ} \mathrm{C}$, affording mainly the desired product $5 \mathrm{a}$ albeit with the poor yield ( $21 \%$ over three steps) and moderate enantiomeric excesses (ee) (51\%) (Table 1, entry 1). Encouraged by this result, the effect of additives was then investigated (for details, see Supplementary Table 1). The presence of a basic additive was found to be crucial to improve the reaction outcome, with $\mathrm{Et}_{3} \mathrm{~N}$ giving the best result (entry 4), the main byproducts were the decomposition of the amino aldehyde in the second step of acylation and the byproducts of aldol condensation have not been detected. In contrast, the acid additives gave poor results and reversed the chirality of the resulting products (entries 2-3 vs. 4-6). Subsequently, decreasing the reaction temperature led to slightly lower yield and ee (entries 7-8). Further examination of the analogous Cat 2-4 revealed that the TfNH modified Cat 1 provided a much better result than Cat 2-4 with the weaker and stronger electron withdrawing property of tolylsulfonyl, $p$-(triflouromethyl)-benzenesulfonyl, and methylsulfonyl, respectively (entries 9-11 vs. 4). In addition, other types of catalysts 
with quite different levels of reactivity and/or stereo-effect from Cat 1 were subjected to the reaction and compared with Cat 1 under the same condition as entry 4 . These include the following: (1) Cat 5, Cat 13, which does not have an acidic functional group; (2) Cat 6, which has a protecting group of pyrrolidine amine; (3) Cat 8, which has a single, stronger acidity and generally identified excellent enatio-induction of BINOL framework; and (4) Cat 9-12 $2^{5,40-44}$, which have similar reactivity but quite different stereo-effect of a linear derivative. Unfortunately, all of these catalysts gave poor results (entries 12-18). Particularly, Cat 7 failed to promote this sequence and only the starting material was recovered (entry 14). These results indicated that Cat 1 exhibited both excellent reactivity and high enantio-induction ability toward the studied transformation.

Substrate scope. Subsequently, the optimal condition of entry 4 (the bold values in table 1) was employed for substrate expansion. First, a variety of substituted 3,4-dihydro- $\beta$-carbolines $\mathbf{2 b}-\mathbf{2 j}$ with a variety of substituents on the indole rings were examined and all reacted smoothly to afford the desired products 5b-5j (Fig. 2a) ${ }^{45-49}$. In detail, the EWG (electron-withdrawing group) substitutions with $\mathrm{F}, \mathrm{Cl}$, and $\mathrm{Br}$ at $\mathrm{C} 9-\mathrm{C} 11$ of the indole ring generally gave high ee (90-92\%) and good yields (51-54\% over three steps) (5d-5h), whereas the EDG (electron-donating group) substitutions gave slightly lower ee $(88 \%$ ee) and yields (45\%) (5b and 5c). Interestingly, the C12 substitutions with either EWG (5i) or EDG (5j) both gave high ee $(94 \%$ and $90 \%$ ee) but merely moderate yields (43\% and $37 \%)$. Moreover, substitutions at the indole nitrogen atom with the EWG (5k) or EDG (5l) resulted in similarly lower ee $(83 \%$ and $76 \%)$ and varied yields (52\% and $32 \%)$. In addition, variation of acyl halides could also generate high ee (both $91 \%$ ee) and moderate yields (45\% and $46 \%$ ) (5m and 5n), respectively. Moreover, changing the Wittig reagents gave moderate yields $(42 \%$ and $40 \%)$ and high ee (both $91 \%$ ee) (5o and 5 p), which were the key intermediates for the synthesis of natural products $\mathbf{1 a / 1 \mathbf { b }}$. Notably, two 3,4-dihydroisoquinoline substrates were explored for the catalytic Mannich/ acylation/Wittig sequence, which also proved to be effective, generating the corresponding products $\mathbf{5 q}$ and $\mathbf{5 r}$ with good ee ( $81 \%$ and $87 \%)$ and moderate yields ( $41 \%$ and $36 \%)$, respectively (Fig. 2b). As a result, the explored Cat 1 could facilitate well the expected asymmetric Mannich sequence with a wide substrate toleration in good to high efficiency in most cases.

Asymmetric total synthesis of naucleofficine I and II. To demonstrate the utility of this sequence, the asymmetric total synthesis of $\mathbf{1 a} / \mathbf{1} \mathbf{b}$ were carried out efficiently from intermediates 5o. First, 5p underwent an $O$-hetero-Diels-Alder cycloaddition under reflux in mesitylene to produce the advanced pentacyclic intermediate 7 with mainly cis-configuration of C3 and C15 (Fig. 3 $)^{50}$. Next, we turned to the asymmetric total synthesis of the natural alkaloids $\mathbf{1 a}$ and $\mathbf{1 b}$ from intermediate $\mathbf{5 0}$, which was readily prepared on the gram-scale from 2 a using $10 \mathrm{~mol} \%(R, R)$ Cat 1 in $42 \%$ yield and $91 \%$ ee (Fig. 4 ). After extensive screening of the reaction conditions (for details, see Supplementary Table 2), $\mathrm{ZnBr}_{2}$ was found to enable the expected $O$-heteroDiels-Alder cyclization of 5o, efficiently affording the pentacyclic intermediate $\mathbf{8}$ with four desired stereocenters in $51 \%$ yield and $>20: 1 \mathrm{dr}$ (Fig. 5), and there were amount of natural products that owned trans-configuration of $\mathrm{C} 3$ and $\mathrm{C} 15$ had not been synthesized. One-recrystallization of $\mathbf{8}$ in $\mathrm{MeOH} / \mathrm{Et}_{2} \mathrm{O}$ enhanced the enantio-purity to $>99 \%$ ee ( $85 \%$ yield) and its absolute configuration was confirmed by X-ray crystallographic analysis (for details, see Supplementary Table 6). However, a thermal cyclization of $\mathbf{5 o}$ in mesitylene under refluxing temperature resulted mainly in the formation of undesired C15-epimer. Hydroformylation of $\mathbf{8}$ with $\mathrm{POCl}_{3} / \mathrm{DMF}$ afforded aldehyde $\mathbf{9}$ in $48 \%$ yield, which was treated with freshly prepared $\mathrm{Me}_{2} \mathrm{CuLi}$ to provide the separable products 10, 11, and 11' (2.8: 2.5: 1) with $63 \%$ total yield. Fortunately, isomer $\mathbf{1 1}^{\prime}$ could be readily converted to $\mathbf{1 1}$ with $85 \%$ yield when stirred in DBU (1,8-diazabicyclo[5.4.0] undec-7-ene)/DCM. Subsequent reduction of 10 with $\mathrm{NaBH}_{4}$ furnished 12 with $86 \%$ yield. After extensive investigations ${ }^{51,52}$, protection of the hydroxyl group of 12 with hexamethyl disilylamine at refluxing temperature gave a silyl ether, which was subjected to elimination of methoxy group and removal of Boc with $\mathrm{BF}_{3} \cdot \mathrm{Et}_{2} \mathrm{O}$ and TMSOTf in $\mathrm{CH}_{3} \mathrm{CN}$ to obtain acetal 14, and then the double bond isomerization to give 1a with $81 \%$ yield, so we developed an efficient method to construct the double bond of these natural products. In the same way, $\mathbf{1 b}$ was also synthesized separately from 11. The spectra data of synthetic (+)-1a and (-)-1b are consistent with literature ${ }^{13}$, and the ee of $(+)-\mathbf{1 a}$ and $(-)-\mathbf{1 b}$ are $>99 \%$ determined by chiral highperformance liquid chromatography (HPLC).

\section{Discussion}

In summary, a chiral bifunctional organocatalyst has been successfully developed, which is a good assembly of strong acid and sterically hindered SPD framework, and thus exhibits high reactivity as well as excellent stereo-induction ability toward the asymmetric Mannich reaction of 3,4-dihydro- $\beta$-carboline with a broad substrate toleration. Furthermore, synthetic utility of this Mannich sequence has been exemplified through the asymmetric total synthesis of naucleofficine I (1a) and II (1) $\mathbf{1})$ in a concise way. Further expanded studies on asymmetric catalysis with our bifunctional organocatalysts toward other transformations and synthesis of other natural products are underway.

\begin{abstract}
Methods
General information. All reactions requiring anhydrous conditions were carried out under argon atmosphere using oven-dried glassware $\left(130^{\circ} \mathrm{C}\right)$, which was cooled under argon. All solvents were purified and dried by standard techniques, and distilled prior to use. All reactions under standard conditions were monitored by thin-layer chromatography (TLC) on gel $\mathrm{F}_{254}$ plates. The products were purified by flash column chromatography on silica gel (200 300 mesh) or neutral alumina (200 300 mesh). ${ }^{1} \mathrm{H}$ NMR, ${ }^{13} \mathrm{C}$ NMR, and ${ }^{19} \mathrm{~F}$ NMR spectra were obtained on Bruker AM-400, JEOL JNM-ECS-400, or Varian Mercury-600. Chemical shifts $(\delta)$ were reported in p.p.m. relative to residual solvent signals $\left(\mathrm{CDCl}_{3}: 7.26\right.$ p.p.m. for ${ }^{1} \mathrm{H}$ NMR, 77.0 p.p.m. for ${ }^{13} \mathrm{C}$ NMR; DMSO- $d_{6}: 2.50$ p.p.m. for ${ }^{1} \mathrm{H}$ NMR, 39.5 p.p. m. for ${ }^{13} \mathrm{C}$ NMR; $\mathrm{CD}_{3} \mathrm{OD}$ : 3.31 p.p.m. for ${ }^{1} \mathrm{H}$ NMR, 49.0 p.p.m. for $\left.{ }^{13} \mathrm{C} N M R\right)$. The following abbreviations were used to indicate the multiplicity in NMR spectra: $s$, singlet; d, doublet; $t$, triplet; $m$, multiplet. High-resolution mass spectral analysis data were measured on the Bruker ApexII with ESI resource. Infrared spectra were recorded on Nicolet FT-170SX spectrometer. Melting points were measured on a melting point apparatus and were uncorrected. The ee of the products were determined by HPLC analysis. X-ray diffraction data were collected on Agilent SuperNova Eos diffractometer. Optical rotations were detected on RUDOLPH A21202-J APTV/GW.
\end{abstract}

General procedure for the Mannich sequent reaction. To a solution of substituted 3,4-dihydro- $\beta$-carboline $2(0.1 \mathrm{mmol})$, Cat $1(5.4 \mathrm{mg}, 0.02 \mathrm{mmol})$ in DCE/ $\mathrm{H}_{2} \mathrm{O}(1 \mathrm{~mL}, v / v 1: 1)$ were added $\mathrm{Et}_{3} \mathrm{~N}(7 \mu \mathrm{L}, 0.05 \mathrm{mmol})$ and aldehyde $(16 \mu \mathrm{L}$, $0.3 \mathrm{mmol}$ ) at $0{ }^{\circ} \mathrm{C}$. The mixture was stirred at this temperature until the substrate disappeared via TLC detection. Then the resulting solution was concentrated in vacuo. Then $1 \mathrm{~mL} \mathrm{DCM}, \mathrm{K}_{2} \mathrm{CO}_{3}(27.6 \mathrm{mg}, 0.2 \mathrm{mmol})$, and acetyl bromide $3 \mathrm{a}$ $(10 \mu \mathrm{L}, 0.12 \mathrm{mmol})$ were added at $0{ }^{\circ} \mathrm{C}$ sequentially. About 30 min later, $4 a$ $(66.8 \mathrm{mg}, 0.2 \mathrm{mmol})$ was added to the reaction at room temperature and reacted at the same temperature for $12 \mathrm{~h}$. The mixture was then quenched with water and the mixture was extracted with DCM. Then the combined organic phase was washed with saturated brine, dried over anhydrous $\mathrm{Na}_{2} \mathrm{SO}_{4}$, and concentrated in vacuo. The residue was purified through column chromatography on neutral alumina to give substrates 5 . 
a
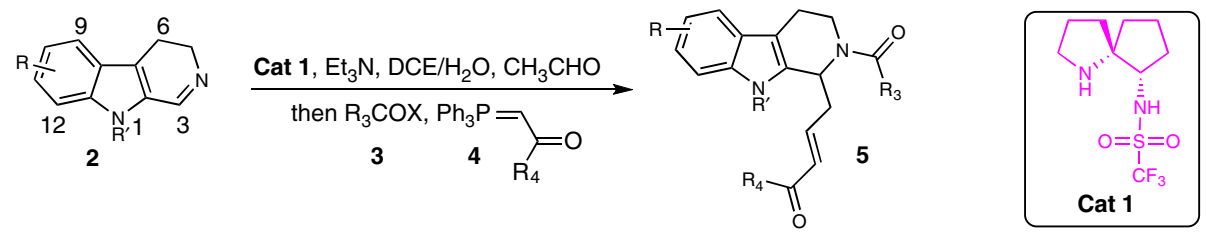

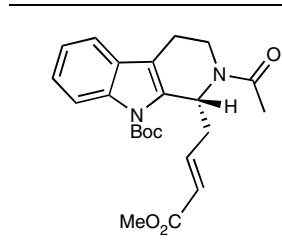

$51 \%$ yield, $91 \%$ ee

$5 a$

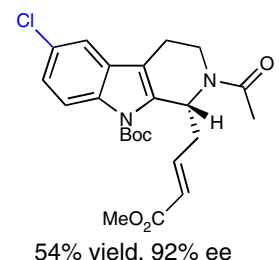

$54 \%$ yield, $92 \%$ ee

$5 e$<smiles>CC(=O)/C=C/C[C@H]1c2[nH]c3c(Br)cccc3c2CCN1C(C)=O</smiles>

$43 \%$ yield, $94 \%$ ee

$5 \mathbf{i}$

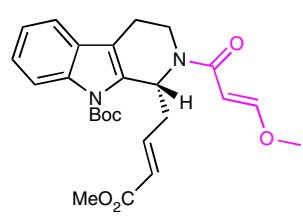

$45 \%$ yield, $91 \%$ ee

$5 \mathrm{~m}$

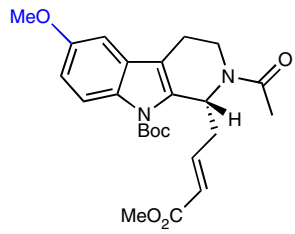

$45 \%$ yield, $88 \%$ ee

$5 b$

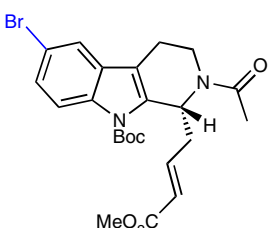

$53 \%$ yield, $92 \%$ ee

$5 f$

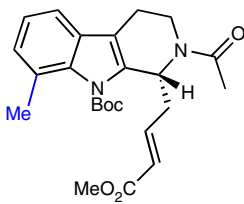

$37 \%$ yield, $90 \%$ ee

5j

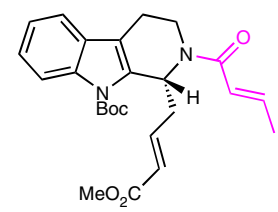

$46 \%$ yield, $91 \%$ ee

$5 n$
$\mathrm{Me}$<smiles>CC(=O)/C=C/C[C@H]1c2[nH]c3ccc(F)cc3c2CCN1C(C)=O</smiles>

$45 \%$ yield, $88 \%$ ee

$5 \mathrm{c}$<smiles></smiles>

$51 \%$ yield, $91 \%$ ee

$5 \mathrm{~g}$

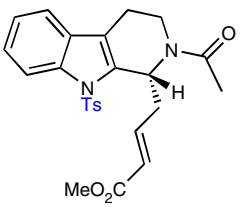

$52 \%$ yield, $83 \%$ ee

$5 k$

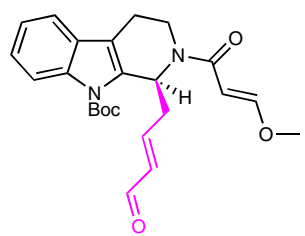

$42 \%$ yield, $91 \%$ ee

50

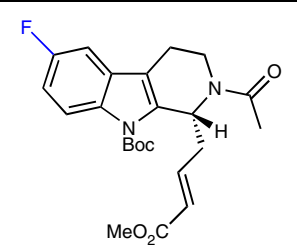

$52 \%$ yield, $90 \%$ ee

$5 d$<smiles>CC(=O)/C=C/C[C@H]1c2[nH]c3cc(F)ccc3c2CCN1C(C)=O</smiles>

$52 \%$ yield, $91 \%$ ee

$5 \mathrm{~h}$

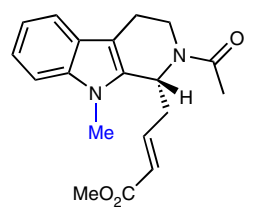

$32 \%$ yield, $76 \%$ ee

51

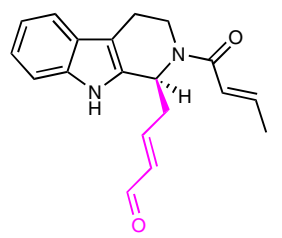

$40 \%$ yield, $91 \%$ ee

$5 p$

b<smiles>[X]c1cccc2c1CCN=C2</smiles>

2
Cat 1, $\mathrm{Et}_{3} \mathrm{~N}, \mathrm{DCE} / \mathrm{H}_{2} \mathrm{O}, \mathrm{CH}_{3} \mathrm{CHO}$

then $\mathrm{R}_{3} \mathrm{COX}, \mathrm{Ph}_{3} \mathrm{P}=$

3

$4 \mathrm{CO}_{2} \mathrm{Me}$

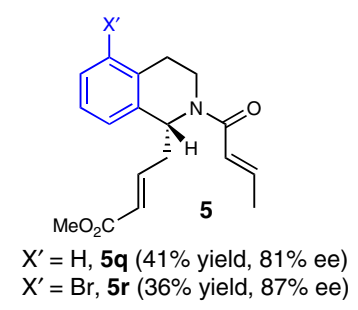

Fig. 2 The substrate scope of the Mannich/Acylation/Wittig reaction. Reaction condition: $\mathbf{2 a}(0.1 \mathrm{mmol})$, acetaldehyde (0.3 mmol), (S,S)-Cat 1 (0.02 $\mathrm{mmol})$, and $\mathrm{Et}_{3} \mathrm{~N}(0.05 \mathrm{mmol})$, in $0.5 \mathrm{~mL} \mathrm{DCE}$ and $0.5 \mathrm{~mL} \mathrm{H} \mathrm{H}_{2} \mathrm{O}$, then acyl halide $\mathbf{3}(0.15 \mathrm{mmol})$ and Wittig reagent 4 (0.2 $\left.\mathrm{mmol}\right)$. Isolated yield over three steps. Enantiomeric excess determined by HPLC analysis. a The scope of 3,4-dihydro- $\beta$-carboline substrates. b The scope of 3,4-dihydro-isoquinoline substrates

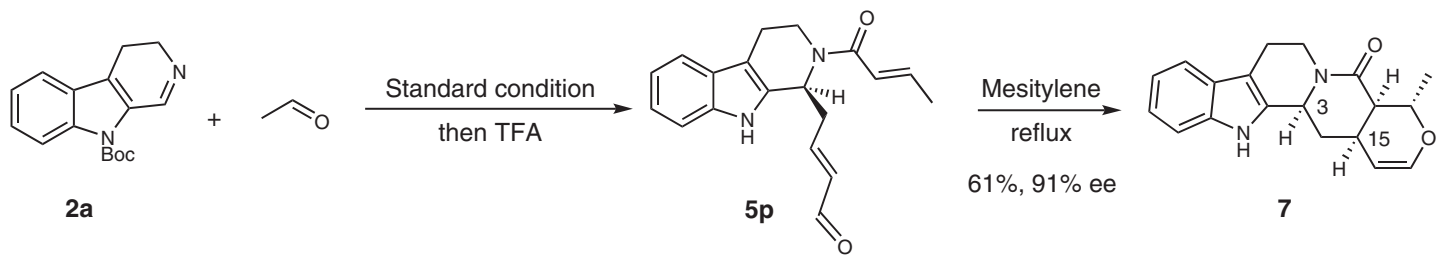

Fig. 3 Further transformation. Thermocatalytic hetero-Diels-Alder reaction mainly obtained the cis-product of $\mathbf{C 3}$ and $\mathbf{C 1 5}$ 


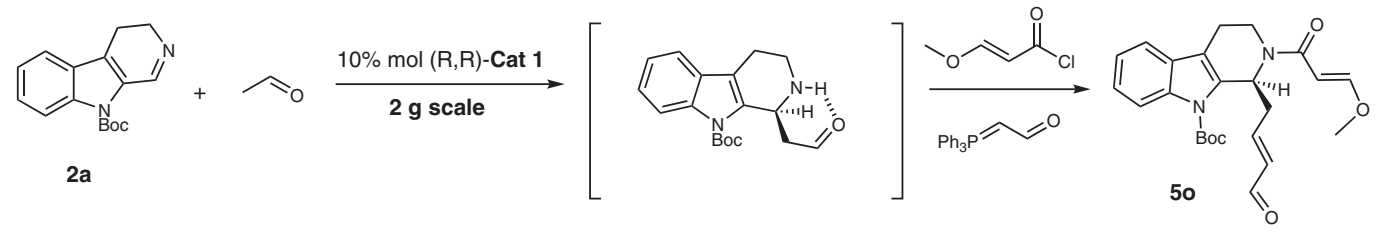

$42 \%$ yield, $91 \%$ ee

Fig. 4 Gram-scale synthesis of $\mathbf{5 0}$. Reactions were performed with $10 \% \mathrm{~mol}(R, R)$-Cat 1

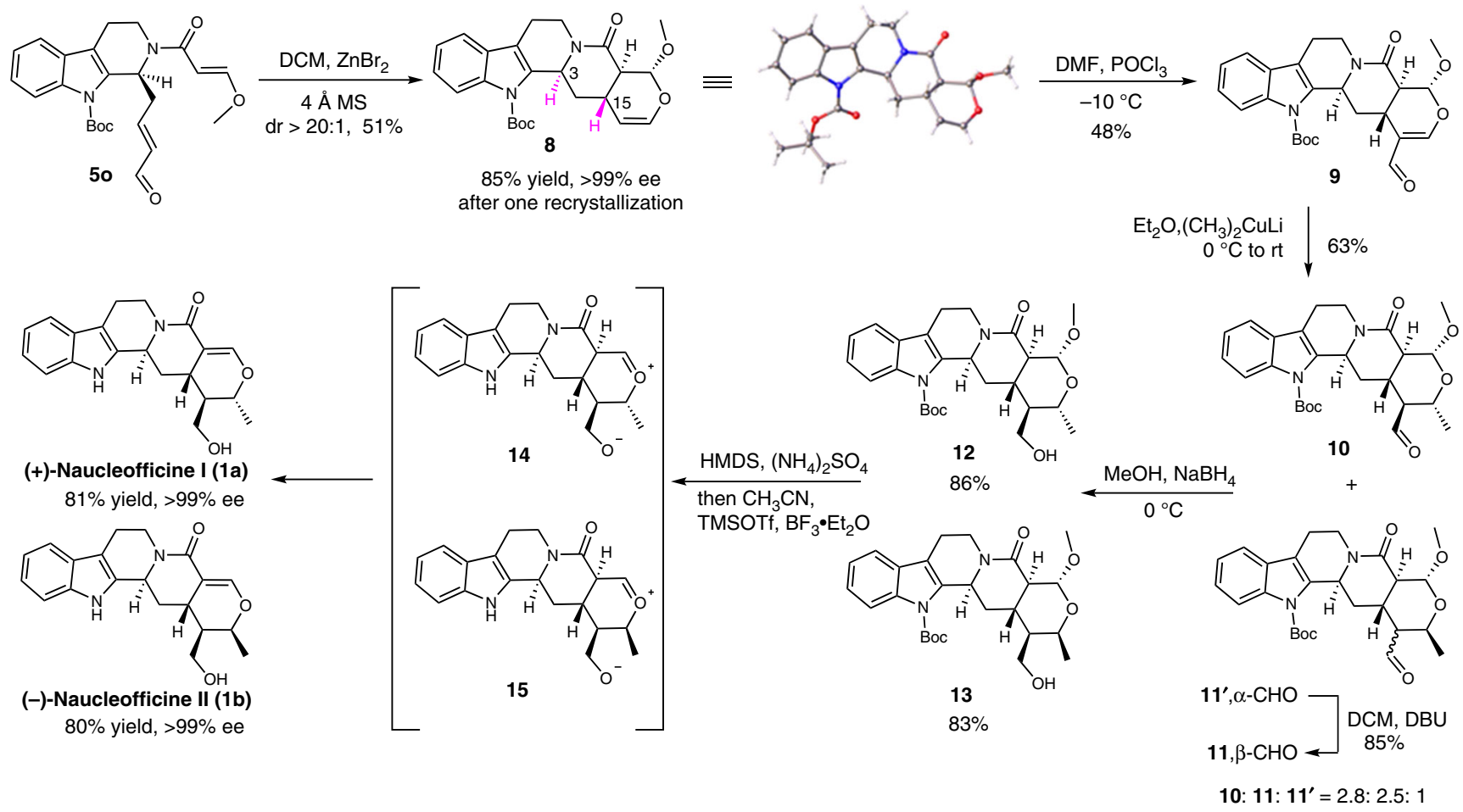

Fig. 5 Asymmetric total synthesis of naucleofficine I and II. The key steps include O-hetero-Diels-Alder cycloaddition, hydroformylation, and double bond isomerization

\section{Data availability}

The X-ray crystallographic coordinates for structures reported in this study have been deposited at the Cambridge Crystallographic Data Centre (Cat 1: CCDC 1875257, 8: CCDC 1875256). These data can be obtained free of charge from The Cambridge Crystallographic Data Centre via www.ccdc.cam.ac.uk/data_request/cif.

Received: 24 April 2019 Accepted: 11 July 2019

Published online: 29 July 2019

\section{References}

1. Ishikawa, H., Suzuki, T. \& Hayashi, Y. High-yielding synthesis of the antiinfluenza neuramidase inhibitor (-)-Oseltamivir by three "One-Pot" operations. Angew. Chem. Int. Ed. 48, 1304-1307 (2009).

2. Jones, S. B., Simmons, B., Mastracchio, A. \& MacMillan, D. W. Collective synthesis of natural productsby means of organocascade catalysis. Nature $\mathbf{4 7 5}$, 183-188 (2011).

3. $\mathrm{Xu}, \mathrm{X}$.-Y. et al. Asymmetric organocatalytic direct aldol reactions of ketones with $\alpha$-keto acids and their application to the synthesis of 2-hydroxy- $\gamma$ butyrolactones. J. Org. Chem. 72, 9905-9913 (2007).

4. Torii, H., Nakadai, M., Ishihara, K., Saito, S. \& Yamamoto, H. Asymmetric direct aldol reaction assisted by water and a proline-derived tetrazole catalyst. Angew. Chem. Int. Ed. 43, 1983-1986 (2004).

5. Albrecht, L., Jiang, H. \& Jørgensen, K. A. Hydrogen-bonding in aminocatalysis: from proline and beyond. Chem. Eur. J. 20, 358-368 (2014).

6. Hajos, Z. G. \& Parrish, D. R. Asymmetric synthesis of bicyclic intermediates of natural product chemistry. J. Org. Chem. 39, 1615-1621 (1974).
7. List, B., Lerner, R. A. \& Barbas, C. F. Proline-catalyzed direct asymmetric aldol reactions. J. Am. Chem. Soc. 122, 2395-2396 (2000).

8. List, B. The direct catalytic asymmetric three-component Mannich reaction. J. Am. Chem. Soc. 122, 9336-9337 (2000).

9. Allemann, C., Gordillo, R., Clemente, F. R., Cheong, P. H.-Y. \& Houk, K. N. Theory of asymmetric organocatalysis of aldol and related reactions: rationalizations and predictions. Acc. Chem. Res. 37, 558-596 (2004).

10. Notz, W., Tanaka, F. \& Barbas, C. F. III Enamine-based organocatalysis with proline and diamines: the development of direct catalytic asymmetric aldol, Mannich, Michael, and Diels-Alder reactions. Acc. Chem. Res. 37, 580-591 (2004).

11. Gruttadauria, M., Giacalone, F. \& Noto, R. Supported proline and prolinederivatives as recyclable organocatalysts. Chem. Soc. Rev. 37, 1666-1688 (2008).

12. Kumar, P. \& Dwivedi, N. Proline catalyzed $\alpha$-aminoxylation reaction in the synthesis of biologically active compounds. Acc. Chem. Res. 46, 289-299 (2013).

13. Wang, H.-Y. et al. Three new isomeric indole alkaloids from Nauclea officinalis. Chem. Biodivers. 12, 1256-1262 (2015).

14. Boucherle, B. et al. Nauclea latifolia: biological activity and alkaloid phytochemistry of a West African tree. Nat. Prod. Rep. 33, 1034-1043 (2016).

15. Mergott, D. J., Zuend, S. J. \& Jacobsen, E. N. Catalytic asymmetric total synthesis of (+)-Yohimbine. Org. Lett. 10, 745-748 (2008).

16. Lebold, T. P. et al. A divergent approach to the synthesis of the yohimbinoid alkaloids venenatine and alstovenine. Nat. Chem. 5, 126-131 (2013).

17. Younai, A., Zeng, B. S., Meltzer, H. Y. \& Scheidt, K. A. Enantioselective syntheses of heteroyohimbine natural products: a unified approach through cooperative catalysis. Angew. Chem. Int. Ed. 54, 6900-6904 (2015).

18. Wang, X. et al. A radical cascade enabling collective syntheses of natural products. Chem 2, 803-816 (2017). 
19. Tian, J.-M. et al. The design of a spiro-pyrrolidine organocatalyst and its application to catalytic asymmetric Michael addition for the construction of all-carbon quaternary centers. Chem. Commun. 51, 9979-9982 (2015).

20. Tian, J.-M. et al. Catalytic asymmetric cascade using spiro-pyrrolidine organocatalyst: efficient construction of hydrophenanthridine derivatives. Org. Lett. 19, 6618-6621 (2017).

21. Chen, S.-K. et al. Organo-cation catalyzed asymmetric homo/ heterodialkylation of bisoxindoles: construction of vicinal all-carbon quaternary stereocenters and total synthesis of (-)-chimonanthidine. J. Am. Chem. Soc. 140, 10099-10103 (2018).

22. Nakashima, D. \& Yamamoto, H. Design of chiral $N$-triflyl phosphoramide as a strong chiral Brønsted acid and its application to asymmetric Diels-Alder reaction. J. Am. Chem. Soc. 128, 9626-9627 (2006).

23. Han, Z.-Y. et al. Hybrid metal/organo relay catalysis enables enynes to be latent dienes for asymmetric Diels-Alder reaction. J. Am. Chem. Soc. 134, 6532-6535 (2012).

24. Wang, Q., van Gemmeren, M. \& List, B. Asymmetric disulfonimide-catalyzed synthesis of $\delta$-amino- $\beta$-ketoester derivatives by vinylogous

Mukaiyama-Mannich reactions. Angew. Chem. Int. Ed. 53, 13592-13595 (2014).

25. Zhou, F. \& Yamamoto, H. A disulfonimide catalyst for highly enantioselective Mukaiyama-Mannich reaction. Org. Lett. 18, 4974-4977 (2016).

26. Trost, B. M., Saget, T. \& Hung, C. I. Direct catalytic asymmetric Mannich reactions for the construction of quaternary carbon stereocenters. J. Am. Chem. Soc. 138, 3659-3662 (2016).

27. Dai, J. et al. Chiral primary amine catalysis for asymmetric Mannich reactions of aldehydes with ketimines: stereoselectivity and reactivity. Angew. Chem. Int. Ed. 56, 12697-12701 (2017).

28. Qiao, B., Huang, Y. J., Nie, J. \& Ma, J.-A. Highly regio-, diastereo-, and enantioselective Mannich reaction of allylic ketones and cyclic ketimines: access to chiral Benzosultam. Org. Lett. 17, 4608-4611 (2015).

29. Arrayas, R. G. \& Carretero, J. C. Catalytic asymmetric direct Mannich reaction: a powerful tool for the synthesis of $\alpha, \beta$-diamino acids. Chem. Soc. Rev. 38, 1940-1948 (2009).

30. Jiang, Y. \& Schaus, S. E. Asymmetric Petasis Borono-Mannich allylation reactions catalyzed by chiral biphenols. Angew. Chem. Int. Ed. 56, 1544-1548 (2017).

31. Verkade, J. M., van Hemert, L. J., Quaedflieg, P. J. \& Rutjes, F. P. Organocatalysed asymmetric Mannich reactions. Chem. Soc. Rev. 37, 29-41 (2008).

32. Trost, B. M., Gnanamani, E., Tracy, J. S. \& Kalnmals, C. A. Zn-ProPhenol catalyzed enantio- and diastereoselective direct vinylogous Mannich reactions between $\alpha, \beta$ - and $\beta, \gamma$-butenolides and aldimines. J. Am. Chem. Soc. 139, 18198-18201 (2017).

33. Yang, J. W., Chandler, C., Stadler, M., Kampen, D. \& List, B. Proline-catalysed Mannich reactions of acetaldehyde. Nature 452, 453-455 (2008).

34. García-García, P., Ladépêche, A., Halder, R. \& List, B. Catalytic Asymmetric Michael reactions of acetaldehyde. Angew. Chem., Int. Ed. 47, 4719-4721 (2008).

35. Hayashi, Y., Itoh, T., Aratake, S. \& Ishikawa, H. A diarylprolinol in an asymmetric, catalytic, and direct crossed-aldol reaction of acetaldehyde. Angew. Chem. Int. Ed. 47, 2082-2084 (2008).

36. Hayashi, Y., Itoh, T., Ohkubo, M. \& Ishikawa, H. Asymmetric Michael reaction of acetaldehyde catalyzed by diphenylprolinol silyl ether. Angew. Chem. Int. Ed. 47, 4722-4724 (2008)

37. Hayashi, Y. et al. Direct organocatalytic Mannich reaction of acetaldehyde: an improved catalyst and mechanistic insight from a computational study. Angew. Chem. Int. Ed. 47, 9053-9058 (2008).

38. Kim, S. M., Kim, Y. S., Kim, D. W., Rios, R. \& Yang, J. W. Acetaldehyde: a small organic molecule with big impact on organocatalytic reactions. Chem. Eur. J. 22, 22 14-2234 (2016).

39. Itoh, T., Ishikawa, H. \& Hayashi, Y. Asymmetric aldol reaction of acetaldehyde and isatin derivatives for the total syntheses of entConvolutamydine E and CPC-1 and a half fragment of Madindoline A and B. Org. Lett. 11, 3854-3857 (2009).

40. Wang, W., Wang, J. \& Li, H. Direct, highly enantioselective pyrrolidine sulfonamide catalyzed Michael addition of aldehydes to nitrostyrenes. Angew. Chem. Int. Ed. 44, 1369-1371 (2005).

41. Wang, J. et al. Enantio- and diastereoselective Michael addition reactions of unmodified aldehydes and ketones with nitroolefins catalyzed by a pyrrolidine sulfonamide. Chem. Eur. J. 12, 4321-4332 (2006)

42. Zu, L., Wang, J., Li, H. \& Wang, W. A recyclable fluorous (S)-pyrrolidine sulfonamide promoted direct, highly enantioselective Michael addition of ketones and aldehydes to nitroolefins in water. Org. Lett. 8, 3077-3079 (2006).

43. Cao, C.-L., Sun, X.-L., Kang, Y.-B. \& Tang, Y. Enantioselective formal [3+3] annulation for the direct construction of bicyclic skeletons with four stereogenic centers. Org. Lett. 9, 4151-4154 (2007).
44. Miura, T. et al. Direct asymmetric aldol reaction with recyclable fluorous organocatalyst. Org. Lett. 12, 1620-1623 (2010).

45. Itoh, T. et al. Preparation of both enantiomers of 1-allyl-1,2,3,4-tetrahydro- $\beta$ carboline using allyltin reagents and a chiral auxiliary derived from L-proline. Tetrahedron 57, 7277-7289 (2001).

46. Itoh, T., Yokoya, M., Miyauchi, K., Nagata, K. \& Ohsawa, A. Proline-catalyzed asymmetric addition reaction of 9 -Tosyl-3,4-dihydro- $\beta$-carboline with ketones. Org. Lett. 5, 4301-4304 (2003).

47. Rajapaksa, N. S., McGowan, M. A., Rienzo, M. \& Jacobsen, E. N. Enantioselective total synthesis of (+)-Reserpine. Org. Lett. 15, 706-709 (2013).

48. Lalonde, M. P., McGowan, M. A., Rajapaksa, N. S. \& Jacobsen, E. N. Enantioselective formal Aza-Diels-Alder reactions of enones with cyclic imines catalyzed by primary aminothioureas. J. Am. Chem. Soc. 135 1891-1894 (2013).

49. Eschenbrenner-Lux, V., Kuchler, P., Ziegler, S., Kumar, K. \& Waldmann, H. An Enantioselective inverse-electron-demand imino Diels-Alder reaction. Angew. Chem. Int. Ed. 53, 2134-2137 (2014).

50. Martin, S. F., Hunter, J. E., Benage, B., Geraci, L. S. \& Mortimore, M. Unified strategy for synthesis of indole and 2-oxindole alkaloids. J. Am. Chem. Soc. 113, 6161-6171 (1991).

51. Yuan, C. \& Hollingsworth, R. I. A short and efficient synthesis of 1,5-anhydrod-glucitol 6-phosphate. Tetrahedron Lett. 52, 5421-5423 (2011).

52. Yoo, S. J., Kim, H. O., Lim, Y. H., Kim, J. M. \& Jeong, L. S. Synthesis of novel $(2 R, 4 R)$ - and $(2 S, 4 S)$-iso dideoxynucleosides with exocyclic methylene as potential antiviral agents. Bioorg. Med. Chem. 10, 215-226 (2002).

\section{Acknowledgements}

We thank the National Natural Science Foundation of China (Nos. 21502080, 21772071 21871117, 21772076), National Science and Technology Major project of the Ministry of Science and Technology of China (2018ZX09711001-005-002), and the '111' Program of MOE for their financial support.

\section{Author contributions}

Y.-H.Y. performed all of experiments and prepared the supplementary information. X.H. and X.G. prepared materials for the synthesis of naucleofficine I and II. X.H. prepared substrates for substrate expansion. F.-P.Z. synthesized Cat 1. J.-M.T. helped the design of Mannich sequent reaction. Y.-Q.T., F.-M.Z., X.-M.Z. and S.-H.W. directed the project and discussed the results. Y.-Q.T. wrote the manuscript.

\section{Additional information}

Supplementary Information accompanies this paper at https://doi.org/10.1038/s41467019-11382-8.

Competing interests: The authors declare no competing interests.

Reprints and permission information is available online at http://npg.nature.com/ reprintsandpermissions/

Peer review information: Nature Communications thanks Alakesh Bisai, Takashi Ohshima and other anonymous reviewer(s) for their contribution to the peer review of this work. Peer reviewer reports are available.

Publisher's note: Springer Nature remains neutral with regard to jurisdictional claims in published maps and institutional affiliations.

Open Access This article is licensed under a Creative Commons Attribution 4.0 International License, which permits use, sharing, adaptation, distribution and reproduction in any medium or format, as long as you give appropriate credit to the original author(s) and the source, provide a link to the Creative Commons license, and indicate if changes were made. The images or other third party material in this article are included in the article's Creative Commons license, unless indicated otherwise in a credit line to the material. If material is not included in the article's Creative Commons license and your intended use is not permitted by statutory regulation or exceeds the permitted use, you will need to obtain permission directly from the copyright holder. To view a copy of this license, visit http://creativecommons.org/ licenses/by/4.0/

(c) The Author(s) 2019 